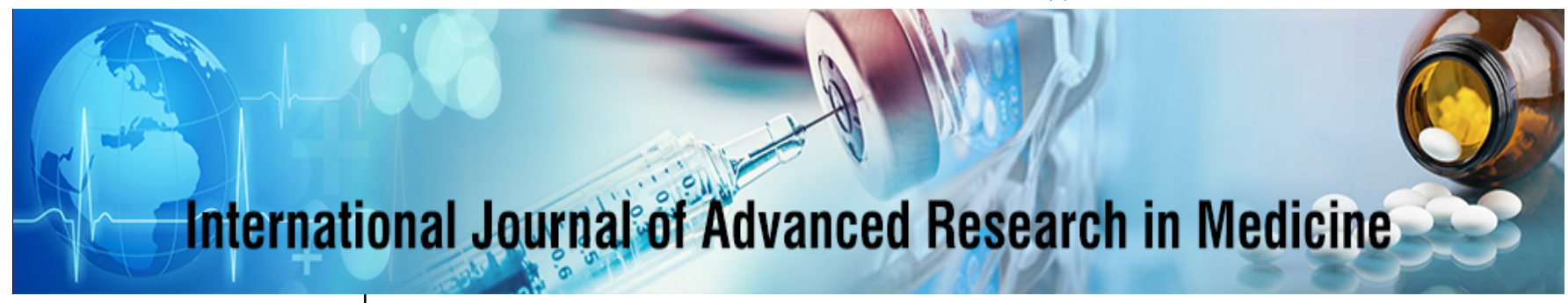

E-ISSN: 2706-9575

P-ISSN: 2706-9567

IJARM 2021; 3(2): 203-208

Received: 07-05-2021

Accepted: 09-06-2021

Dr. Ch Amulya

Senior Resident, Department of Neurology, JSS Medical

College\& Hospitals, Mysuru, Karnataka, India

\section{Dr. Harsha S}

Professor and HOD,

Department of Neurology, JSS

Medical College \& Hospitals,

Mysuru, Karnataka, India

\section{Dr. Nemichandra SC}

Associate Professor,

Department of Neurology, JSS

Medical College \& Hospitals,

Mysuru, Karnataka, India

\section{Dr. Shastara $\mathbf{P}$}

Assistant Professor,

Department of Neurology, JSS

Medical College \& Hospitals,

Mysuru, Karnataka, India
Corresponding Author:

Dr. Ch Amulya

Senior Resident, Department

of Neurology, JSS Medical

College\& Hospitals, Mysuru,

Karnataka, India

\section{Association of anxiety and depression in patients with chronic migraine}

\author{
Dr. Ch Amulya, Dr. Harsha S, Dr. Nemichandra SC and Dr. Shastara P
}

DOI: https://doi.org/10.22271/27069567.2021.v3.i2d.241

\begin{abstract}
Aim: To study the association of depression and anxiety in patients with chronic migraine leading to disability.

Materials and methods: The index cases were obtained from patients attending the Neurology outpatient Department of JSS Hospital, who satisfied International Classification of Headache Disorder 3 beta criteria for diagnosis of chronic migraine. Patients were subjected to Hamilton depression and anxiety rating scales along with, Migraine Disability Assessment Questionnaire (MIDAS) for assessing disability.

Results: A total of 60 patients were studied. The associated anxiety and depression symptoms were more prevalent in patients with chronic migraine which increases disability. As per MIDAS disability assessment 20\% $(n=12)$ had grade I disability, 13.3\% $(n=8)$ had grade II disability, $50 \%(n=30)$ had moderate disability and $16.7 \%(n=10)$ had severe disability.

Conclusion: More prevalence of anxiety and depression was noted in patients with chronic migraine compared to international literature because of variations in study population. Treating co-existing depression and anxiety in patients with chronic migraine decreases disability and also progression to chronic migraine in patients with episodic migraine.
\end{abstract}

Keywords: anxiety, depression in patients, chronic migraine

\section{Introduction}

Headache disorders are very common worldwide. As per the Global Burden of Disease Study 2010 (GBD2010), tension-type headache (TTH) and migraine are considered to be the second and third respectively as the most prevalent disorders in the world ${ }^{[1]}$.

Migraine, a common, neurological disorder and its prevalence is between $5 \%$ and $12 \%$ in the general population ${ }^{[2]}$.

Migraine is common among the general population with estimated prevalence of $6-28 \%$ in women and $2-19 \%$ in men and therefore this would suggest a significant level of morbidity. Migraine symptoms and associated psychiatric disorders are disabling and they interfere with the individual's performance in educational and occupational pursuits and household chores [3]. The WHO has recognized migraine as an urgent public health priority and listed it as the $7^{\text {th }}$ leading cause of disability ${ }^{[4]}$. Migraine is comorbid with a number of psychiatric disorders such as anxiety and depression ${ }^{[5]}$.

Headache frequency and impact of headache are more pronounced in migraine with comorbid psychiatric disorders than in migraine without comorbid psychiatric disorders ${ }^{[6,7 \text {, }}$ 8]. Psychiatric comorbidities are more prevalent in chronic migraine compared to episodic migraine ${ }^{[9]}$. Anxiety is the most common psychiatric comorbidity among patients with migraine, showing a prevalence of $25.5-57.6 \%$ in population-based studies ${ }^{[10]}$.

The Diagnostic and Statistical Manual of Mental Disorders-4 text revision (DSM-IV TR) identified anxiety as an Axis-1 disorder that represented acute symptoms requiring treatment [11].

Patients having migraine with anxiety are more likely to experience disability and poor quality of life compared to those without anxiety ${ }^{[12]}$.

Depression is another Axis-1 disorder in the DSM-IV TR. A close association between depression and migraine has been reported in clinical settings and population-based studies [10].

Depression may exacerbate the impact of migraine and complicate treatment ${ }^{[13,14]}$. 
Longitudinal studies have reported bidirectional comorbidity in which migraine predisposes patients to depression and vice versa ${ }^{[15]}$.

Depression is also shown associated with the transformation from episodic to chronic migraine ${ }^{[16]}$. In many clinical and epidemiological studies, although anxiety and depression have been classified as distinct traits of affective disorders, concurrence of anxiety and depression is observed ${ }^{[17,18]}$.

Most individuals with anxiety or depression possess these traits in mixed form, rather than having pure anxiety or pure depression (Pure affective disorder).

The concurrence of anxiety and depression in migraine has been reported; $42.1-84.6 \%$ of patients having migraine with depression also experience anxiety, and $66.1-85.7 \%$ of patients having migraine with anxiety also have depression. ${ }^{7}$ Major depression was present in $8.6 \%-47.9 \%$ of individuals with migraine in a meta-analytic study, while one study found migraineurs to be 4 or 5 times more likely to suffer from generalized anxiety disorder. However, the comorbidity of anxiety and depression in migraineurs and its clinical significance has rarely been reported. It is essential to identify the patients with comorbid anxiety and depression symptoms, as they are risk factors for conversion of episodic migraine to chronic migraine.

Associated comorbid depression and anxiety are usually associated with poorer long-term headache outcomes, higher medical costs, healthcare utilization, and increased headache-related disability. We hypothesize that anxiety and depression is more common in migraineurs causing more disability than general population.

The present study was conducted to assess the anxiety and depression symptoms in patients with migraine.

\section{Materials and Methods}

The index cases were obtained from patients attending the Neurology Outpatient Department of Neurology, JSS Hospital during the period from December 01, 2020 to July, 2021. The cases were identified to have chronic migraine by International Classification of Headache Disorder 3 beta criteria.

\section{Migraine without aura}

Recurrent headache disorder manifesting in attacks lasting 4- 72 hours. Typical characteristics of the headache are unilateral location, pulsating quality, moderate or severe intensity, aggravation by routine physical activity and association with nausea and/ or photophobia and phonophobia.

\section{Diagnostic criteria for Migraine}

A. At least five attacks fulfilling criteria B-D

B. Headache attacks lasting 4-72 hr (untreated or unsuccessfully treated)

C. Headache has at least two of the following four characteristics:

1. unilateral location

2. pulsating quality

3. moderate or severe pain intensity

4. aggravation by or causing avoidance of routine physical activity (eg, walking or climbing stairs)

D. During headache at least one of the following:

5. Nausea and/or vomiting

6. 2.Photophobia and phonophobia

E. Not better accounted for by another ICHD-3 diagnosis.
Migraine with aura Description:

Recurrent attacks, lasting minutes, of unilateral fullyreversible visual, sensory or other central nervous system symptoms that usually develop gradually and are usually followed by headache and associated migraine symptoms.

Diagnostic criteria:

A. At least two attacks fulfilling criteria B and C

B. One or more of the following fully reversible aura symptoms:

1. Visual

2. Sensory

3. Speech and/or language

4. Motor

5. Brainstem

6. Retinal

C. At least three of the following six characteristics:

1. At least one aura symptom spreads gradually over $\geq 5$ minutes

2. Two or more aura symptoms occur in succession

3. Each individual aura symptom lasts 5-60 minutes

4. 4.At least one aura symptom is unilateral

5. At least one aura symptom is positive

6. The aura is accompanied, or followed within 60 minutes, by headache

D. Not better accounted for by another ICHD-3 diagnosis.

\section{Chronic migraine}

Description: Headache occurring on 15 or more days/month for more than 3 months, which, on at least 8 days/month, has the features of migraine headache.

\section{Diagnostic criteria}

A. Headache (migraine-like or tension-type-like) on $\geq 15$ days/month for $>3$ months, and fulfilling criteria B and C

B. Occurring in a patient who has had at least five attacks fulfilling criteria B-D for 1.1 Migraine without aura and/or criteria B and C for 1.2 Migraine with aura

C. On $\geq 8$ days/month for $>3$ months, fulfilling any of the following

1. criteria $\mathrm{C}$ and $\mathrm{D}$ for 1.1 Migraine without aura

2. criteria B and C for 1.2 Migraine with aura

3. believed by the patient to be migraine at onset and relieved by a triptan or ergot derivative

D. Not better accounted for by another ICHD-3 diagnosis.

Each patient was evaluated in detail using a questionnaire with details regarding the duration of headache, frequency and duration of each episode, site, quality and severity of pain, auras, migraine accompaniments such as photophobia or phonophobia, nausea, vomiting as well as triggers. To assess disability, Migraine Disability Assessment Questionnaire (MIDAS) was applied to all patients. The MIDAS is a well-validated method and consists of five questions to be answered by the patient about the impact of migraine headaches in the past 3 months on their personal, professional and social lives as well another section regarding severity and frequency of these headaches. ${ }^{19}$

A score of 6 or more is taken as positive with cut-offs for mild, moderate, and severe disability. The presence of concurrent anxiety and depression was assessed by the Hamilton Anxiety Rating Scale (HAM-A) and Hamilton Depression Rating Scale (HDRS) respectively. 
HAM-A scale consists of 14 items, each defined by a series of symptoms, and measures both psychic anxiety (mental agitation and psychological distress) and somatic anxiety (physical complaints related to anxiety). Each item is scored on a scale of 0 (not present) to 4 (severe), with a total score range of $0-56$, where $<17$ indicates mild severity, 18-24 mild to moderate severity and 25-30 moderate to severe. HDRS contains 17 items (HDRS17) pertaining to symptoms of depression experienced over the past week. A score of 0 7 is generally accepted to be within the normal range (or in clinical remission), while a score of 20 or higher (indicating at least moderate severity) is usually considered significant. Neuro-imaging studies (magnetic resonance imaging) were done in all patients to rule out structural lesions.

Exclusion criteria were:

1. Presence of other headaches, both secondary and primary, is including tension type headache.

2. Recent onset of headaches within the past 6 months of study inclusion

3. Recent use of hormonal contraceptives

4. Individuals with other medical conditions

5. Individuals previously diagnosed to have psychiatric disease

\section{Results}

A total of 60 patients were studied during the study. Incidence is higher in female patients $(n=37,61.7 \%)$ than male patients $(n=23,38.3 \%)$.

Table 1: Gender distribution

\begin{tabular}{|c|c|c|}
\hline Gender & No. & Percentage \\
\hline Male & 23 & $38.3 \%$ \\
\hline Female & 37 & $61.7 \%$ \\
\hline Total & 60 & $100.0 \%$ \\
\hline
\end{tabular}

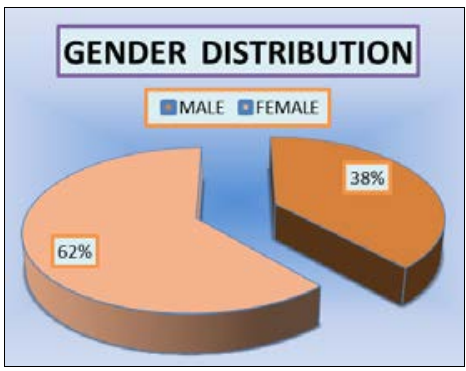

Fig 1: Gender distribution

Maximum patients were within the age group 18-40 $(n=42$, $70 \%$ ) and remaining $30 \% 41-60$ years.

Table 2: Age group distribution

\begin{tabular}{|c|c|c|}
\hline Age group & No. & Percentage \\
\hline $18-40$ yrs & 42 & $70.0 \%$ \\
\hline $41-60$ yrs & 18 & $30.0 \%$ \\
\hline Total & 60 & $100.0 \%$ \\
\hline
\end{tabular}

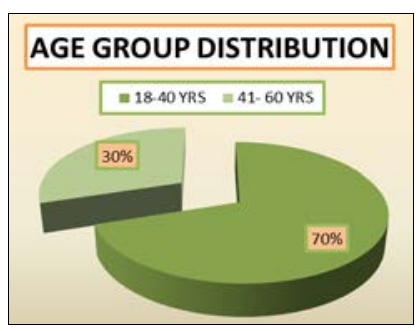

Fig 2: Age group distribution
44 patients (73.3\%) had migraine without aura and 16 patients (26.7\%) had migraine with aura symptoms. 63.3\% $(n=38)$ patients had nausea and vomiting.

Table 3: Associated aura symptoms

\begin{tabular}{|c|c|c|}
\hline Associated aura symptoms & No. & Percentage \\
\hline Without aura symptoms & 44 & $73.3 \%$ \\
\hline With aura symptoms & 16 & $26.7 \%$ \\
\hline Total & 60 & $100.0 \%$ \\
\hline
\end{tabular}

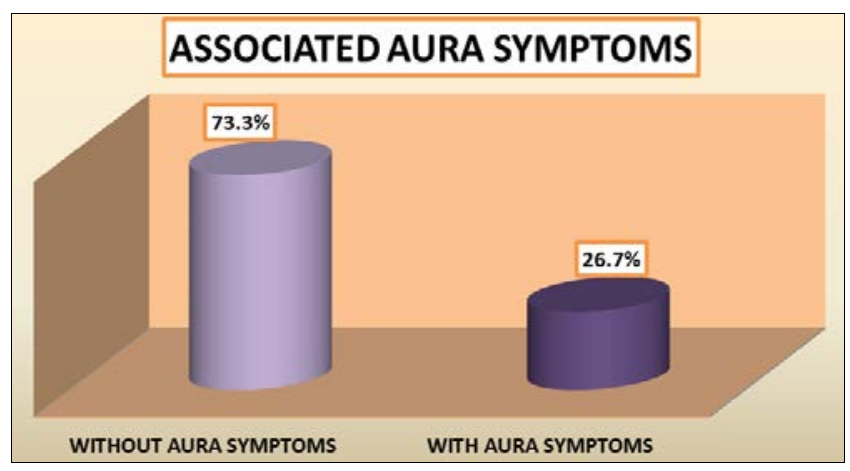

Fig 3: Associated aura symptoms

Table 4: Symptoms

\begin{tabular}{|c|c|c|}
\hline Symptoms & No. & Percentage \\
\hline Headache & 60 & $100.0 \%$ \\
\hline Photophobia \& phonophobia & 46 & $76.7 \%$ \\
\hline Nausea \& vomiting & 38 & $63.3 \%$ \\
\hline Medication over use headache & 14 & $23.3 \%$ \\
\hline
\end{tabular}

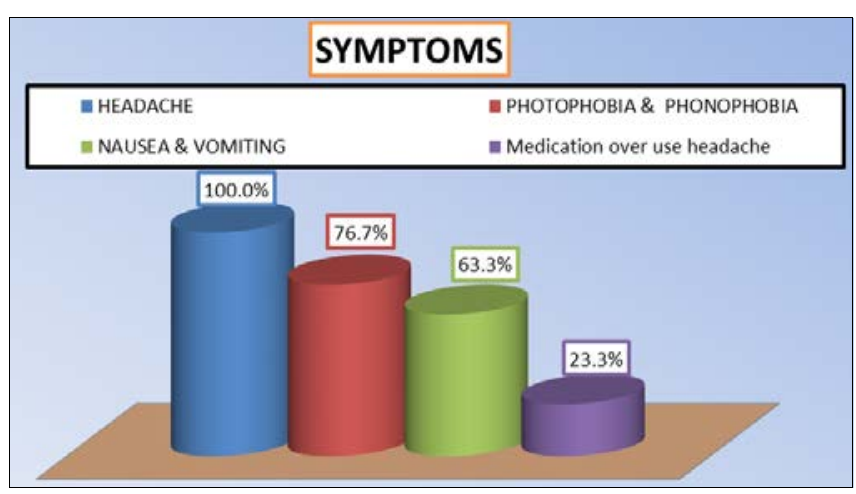

Fig 4: Symptoms

76.7\% $(n=46)$ patients had photophobia and phonophobia. 23.3\% ( $n=14)$ patients had medication over use headache. As per MIDAS disability assessment $20 \%(n=12)$ had grade I disability, $13.3 \%(n=8)$ had grade II disability, 50\% $(n=30)$ had moderate disability and $16.7 \% \quad(n=10)$ had severe disability.

Table 5: Midas criteria for disability

\begin{tabular}{|c|c|c|c|}
\hline Midas criteria for disability & Score & No of patients & Percentage \\
\hline Grade I (no disability) & 0 to 5 & 12 & $20.0 \%$ \\
\hline Grade ii (mild disability) & 6 to 10 & 8 & $13.3 \%$ \\
\hline Grade iii (moderate disability) & 11 to 20 & 30 & $50.0 \%$ \\
\hline Grade iv (severe disability) & $21+$ & 10 & $16.7 \%$ \\
\hline Total & - & 60 & $100.0 \%$ \\
\hline
\end{tabular}




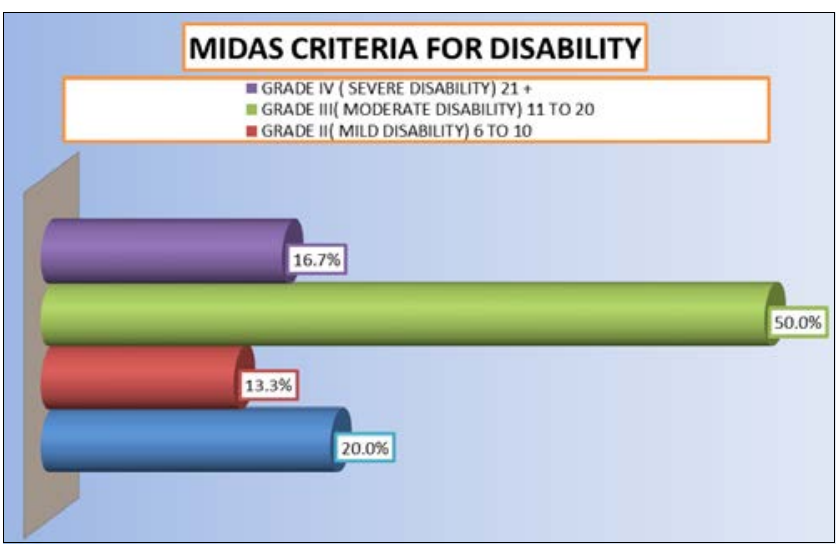

Fig 5: Midas criteria for disability

$66.7 \%$ patients $(n=40)$ had anxiety symptoms of which 24 patients had mild severity $(\leq 17), 12$ patients had mild to moderate severity (18-24), 4 patients had moderate to severe anxiety on HAM-A scale.

Table 6: Anxiety grading (Ham-A scale)

\begin{tabular}{|c|c|c|c|}
\hline Anxiety grading (ham-a scale) & Score & No of patients & Percentage \\
\hline Mild severity & $\leq 17$ & 24 & $40.0 \%$ \\
\hline Mild to moderate severity & $18-24$ & 12 & $20.0 \%$ \\
\hline Severe anxiety & $\geq 25$ & 4 & $6.7 \%$ \\
\hline Total & - & 40 & $66.7 \%$ \\
\hline
\end{tabular}

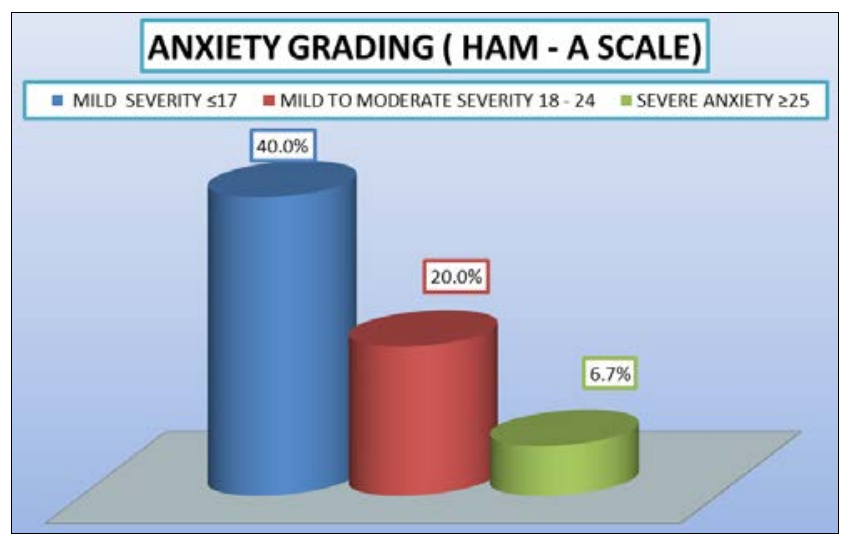

Fug 6: Anxiety grading (Ham-A scale)

$60 \%$ patients $(\mathrm{n}=36)$ had depression symptoms of which 18 patients had mild symptoms (10-13), 12 patients had moderate symptoms (14-17) and 6 patients had severe symptoms $(\geq 17)$ on HDRS scale.

Table 7: Depression grading (HDRS scale)

\begin{tabular}{|c|c|c|c|}
\hline Depression grading (HDRS scale) & Score & No of patients & Percentage \\
\hline Mild symptoms & 10 to 13 & 18 & $50.0 \%$ \\
\hline Moderate & 14 to 16 & 12 & $33.3 \%$ \\
\hline Severe symptoms & $\geq 17$ & 6 & $16.7 \%$ \\
\hline Total & - & 36 & $100.0 \%$ \\
\hline
\end{tabular}

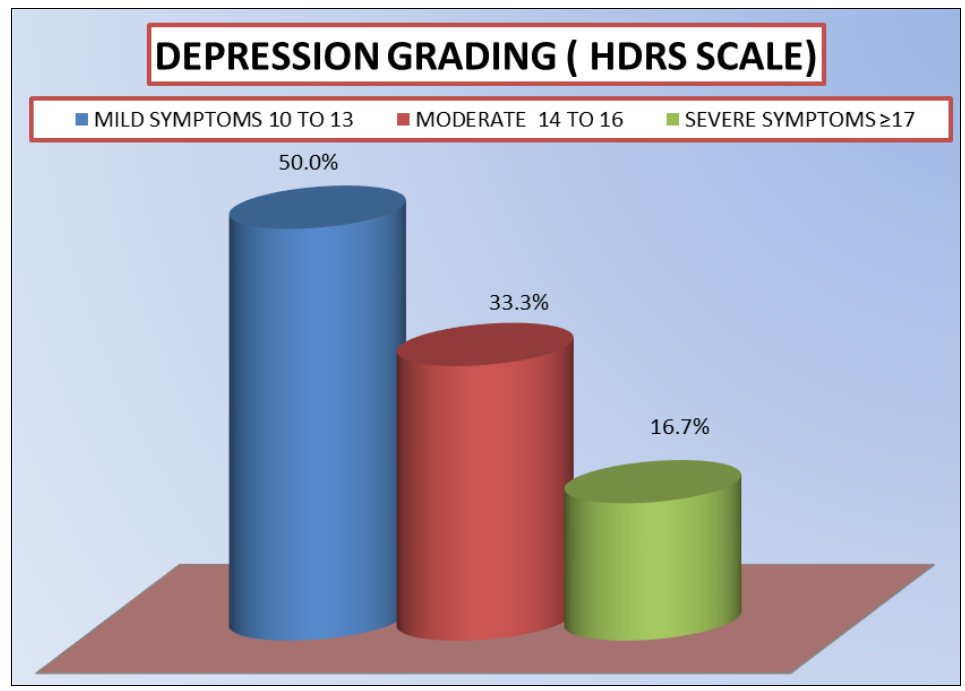

Fig 7: Depression grading (HDRS scale)

\section{Discussion}

Patient's with migraine are 2.5 times more likely to be depressed compared to people without migraine. ${ }^{20}$

Migraine patients tend to experience 2-5 times more anxiety symptoms compared to non migraneurs. Indeed, a few studies have not found an association between migraine and depression. This is likely due to differences in the inclusion criteria (for example the presence of other type of concurrent headaches), clinico-epidemiological variations between different geographic populations as well as differences between the different scales used to demonstrate psychopathology.

Similarly, many studies have confirmed the comorbidity of migraine and anxiety disorders. In fact, the association between migraine and anxiety disorders is even stronger than affective disorders. The majority of migraineurs (51\%$58 \%$ ) will meet criteria for at least one anxiety disorder during their lifetime. Many epidemiological studies indicate that anxiety disorders are nearly twice as common among migraineurs as is depression ${ }^{[21,22,23]}$.

Generalized anxiety disorders and social phobia were the most commonest anxiety disorders associated with migraine.

Several authors have proposed that the onset of anxiety disorders precedes migraine which in turn precedes depression onset.

Analysis of data obtained from our study confirms our hypothesis. $66.7 \%$ patients $(\mathrm{n}=40)$ had anxiety symptoms of which 24 patients had mild severity $(\leq 17), 12$ patients had mild to moderate severity (18-24), 4 patients had moderate to severe anxiety on HAM-A scale and $60 \%$ patients $(n=36)$ had depression symptoms of which 18 patients had mild symptoms (10-13), 12 patients had moderate symptoms (14$17)$ and 6 patients had severe symptoms $(\geq 17)$ on HDRS 
scale, which is correlating with studies from international literature.

In study done by Juliane Prieto Peres Mercante et al., showed Moderate or severe depression, were present in $58.7 \%$ of the patients and some degree of depression appeared in $85.8 \%$ of chronic migraine patients ${ }^{[23]}$.

In the Zurich study, people with migraine had twofoldincreased prevalence of major depression (15\% versus $7 \%$ ). However, little is known about the range of severity of depressive symptoms in chronic migraine patients ${ }^{\text {[24] }}$.

Breslau et al. reported that depression and anxiety co-exist in approximately $30 \%$ of patients with migraine. In their population-based study, $84 \%$ of patients having migraine with depression also had anxiety, and $54 \%$ of patients having migraine with anxiety had depression ${ }^{[25]}$.

Another study from France reported that $84.6 \%$ of patients having migraine with depression had anxiety and $40.4 \%$ of patients having migraine with anxiety had depression ${ }^{[7]}$.

An important finding in this study was that comorbid psychiatric disorders when present, adds significantly to migraine-related disability and if not identified and properly treated leads to unsuccessful headache management.

The relationship between mood disorders and migraine could be multi-factorial. For example, depressive symptoms and anxiety could emerge after recurrent headache episodes or by themselves they could be risk factors for migraine, and finally, they could be associated with some other confounding factor which drives the headache. This has led to the question whether this relationship is unidirectional or bidirectional. In our patients, duration and frequency of headache attacks were found to be more associated with the occurrence of anxiety and depression ${ }^{[16]}$.

Anxiety and depression symptoms are overlapping and should be considered as a continuum of affective symptoms, especially in the presence of pain disorders. In this regard, some studies have found a correlation between the frequency of headaches and occurrence of mood disorders in migraine. Indeed, Baldacci et al. have postulated an interesting template for the etiopathology of comorbid mood disorders based on this. They hypothesized that a combination of increased duration of headache attacks and increased sensory sensitivity in migraineurs, which persists at a lower level in between the attacks is associated with mood disorders.

Hence, in migraine, there is dysmodulation of normal sensory processing leading to a state of cortical hyper excitability for both inherited and acquired causes. They postulate that this hypersensitivity is more pronounced in migraineurs with mood disorders and provide evidence from their study that cutaneous allodynia during headache and higher susceptibility to trigger factors are present more commonly in those individuals with mood disorders ${ }^{[26]}$.

\section{Conclusion}

We found more prevalence of anxiety and depression in patients with chronic migraine compared to international literature because of variations in study population.

Impact of headache increased with the accompaniment of anxiety and/or depression. The presence of anxiety and depression should be carefully evaluated in patients with migraine, in order to reduce the impact of headache as well as provide better treatment for these patients.

Treating co-existing depression and anxiety in patients with chronic migraine decreases disability and facilitates effective management of migraine. Treatment of coexisting mood decreases progression to chronic migraine in patients with episodic migraine.

\section{References}

1. Vos T, Flaxman AD, Naghavi M, Lozano R, Michaud $\mathrm{C}$, Ezzati $\mathrm{M}$ et al. Years lived with disability (YLDs) for 1160 sequelae of 289 diseases and injuries 1990 2010: a systematic analysis for the Global Burden of Disease Study 2010. Lancet 2012;380:2163-2196.

2. Jensen R, Stovner LJ. Epidemiology and comorbidity of headache. Lancet Neurol 2008;7(4):354-361. 10.1016/S1474-4422(08)70062-0.

3. Stovner L, Hagen K, Jensen R, Katsarava Z, Lipton R, Scher A et al. The global burden of headache: a documentation of headache prevalence and disability worldwide. Cephalalgia 2007;27(3):193-210. 10.1111/j.1468-2982.2007.01288.x.

4. Leonardi M, Steiner TJ, Scher A, Lipton R. The global burden of migraine: measuring disability in headache disorders with WHO's Classification of Functioning, Disability and Health (ICF). J Headache Pain 2005;6(6):429-440. 10.1007/s10194-005-0252-4.

5. Breslau N. Psychiatric comorbidity in migraine. Cephalalgia 1998;18(22):56-58. discussion 58

6. Sareen J, Jacobi F, Cox BJ, Belik S-L, Clara I, Stein MB. Disability and poor quality of life associated with comorbid anxiety disorders and physical conditions. Arch Intern Med 2006;166(19):2109-2116. 10.1001/archinte.166.19.2109

7. Lantéri-Minet M, Radat F, Chautard M-H, Lucas C. Anxiety and depression associated with migraine: influence on migraine subjects' disability and quality of life, and acute migraine management. Pain 2005;118(3):319-326. 10.1016/j.pain.2005.09.010.

8. Merikangas KR, Angst J, Isler $H$. Migraine and psychopathology: results of the Zurich cohort study of young adults. Arch Gen Psychiatry 1990;47(9):849853. 10.1001/archpsyc.1990.01810210057008

9. Buse DC, Silberstein SD, Manack AN, Papapetropoulos S, Lipton RB. Psychiatric comorbidities of episodic and chronic migraine. J Neurol 2013;260(8):1960-1969. 10.1007/s00415-0126725-x.

10. McWilliams LA, Goodwin RD, Cox BJ. Depression and anxiety associated with three pain conditions: results from a nationally representative sample. Pain 2004;111(1):77-83. 10.1016/j.pain.2004.06.002.

11. American Psychiatric Association: Diagnostic and statistical manual of mental disorders: DSM-IV-TR ${ }^{\circledR}$, American Psychiatric Pub, Arlington 2000.

12. Zwart JA, Dyb G, Hagen K, Ødegård K, Dahl A, Bovim G et al. Depression and anxiety disorders associated with headache frequency. The NordTrøndelag Health Study. Eur J Neurol 2003;10(2):147152. 10.1046/j.1468-1331.2003.00551.x.

13. Breslau N, Lipton R, Stewart W, Schultz L, Welch K. Comorbidity of migraine and depression investigating potential etiology and prognosis. Neurology 2003;60(8):1308-1312. 10.1212/01.WNL.0000058907.41080.54.

14. Breslau N, Davis GC, Schultz LR, Paterson EL. Migraine and major depression: a longitudinal study. 
Headache 1994;34(7):387-393. 10.1111/j.15264610.1994.hed3407387.x.

15. Haythornthwaite JA. Migraine headaches and psychopathology: Future directions. J Psychiatr Res 1993;27(2):183-186. 10.1016/0022-3956(93)90006-N.

16. Ashina S, Buse DC, Maizels M, Manack A, Serrano D, Turkel C et al. Depression: a risk factor for migraine chronification: results from the American Migraine Prevalence and Prevention (AMPP) study. Neurology 2010;74(2):A113.

17. Kessler RC, Berglund P, Demler O, Jin R, Merikangas $\mathrm{KR}$, Walters EE. Lifetime prevalence and age-of-onset distributions of DSM-IV disorders in the National Comorbidity Survey Replication. Arch Gen Psychiatry 2005;62(6):593-10.1001/archpsyc.62.6.593.

18. Schoevers R, Beekman A, Deeg D, Jonker C, Tilburg WV. Comorbidity and risk-patterns of depression, generalised anxiety disorder and mixed anxiety-depression in later life: results from the AMSTEL study. Int $\mathrm{J}$ Geriatr Psychiatry 2003;18(11):994-1001. 10.1002/gps.1001.

19. Stewart WF, Lipton RB, Dowson AJ, Sawyer J. Development and testing of the migraine disability assessment (MIDAS) questionnaire to assess headacherelated disability. Neurology 2001;56:S20-8.

20. Lipton RB, Hamelsky SW, Kolodner KB, Steiner TJ, Stewart WF. Migraine, quality of life, and depression: A population-based case-control study. Neurology 2000;55:629-35.

21. Devlen J. Anxiety and depression in migraine. J R Soc Med 1994;87:338-41.

22. Merikangas KR, Angst J, Isler $H$. Migraine and psychopathology. Results of the Zurich cohort study of young adults. Arch Gen Psychiatry 1990;47:849-53.

23. Juliane Prieto Peres Mercante et al. Depression in CHRONIC MIGRAINE Severity and clinical features. Arq Neuropsiquiatr 2005;63(2-A):217-220.

24. Buse DC, Manack A, Serrano D, Turkel C, Lipton RB. Sociodemographic and comorbidity profiles of chronic migraine and episodic migraine sufferers. J Neurol Neurosurg Psychiatry 2010;81:428-32.

25. Breslau N, Davis GC, Andreski P. Migraine, psychiatric disorders, and suicide attempts: an epidemiologic study of young adults. Psychiatry Research 1991;37(1):11-23. 10.1016/01651781(91)90102-U.

26. Lakhan SE, Avramut M, Tepper SJ. Structural and functional neuroimaging in migraine: Insights from 3 decades of research. Headache 2013;53:46-66. 